\title{
The pterocarpanquinone LQB-118 induces apoptosis in acute myeloid leukemia cells of distinct molecular subtypes and targets FoxO3a and FoxM1 transcription factors
}

\author{
GABRIELA NESTAL DE MORAES ${ }^{1 *}$, CAROLINA PEREIRA CASTRO ${ }^{1,2^{*}}$, EDUARDO JESUS SALUSTIANO ${ }^{3,4}$, \\ MATHEUS LOURENÇO DUMAS ${ }^{4}$, FERNANDA COSTAS ${ }^{1,2}$, ERIC WING-FAI LAM ${ }^{5}$, \\ PAULO ROBERTO RIBEIRO COSTA ${ }^{3}$ and RAQUEL CIUVALSCHI MAIA ${ }^{1}$
}

\begin{abstract}
${ }^{1}$ Laboratory of Cellular and Molecular Hemato-Oncology, Program of Molecular Hemato-Oncology, ${ }^{2}$ Program of Post-Graduation in Oncology, Brazilian National Cancer Institute (INCA); ${ }^{3}$ Laboratory of Bioorganic Chemistry, Research Nucleous of Natural Products, Federal University of Rio de Janeiro (UFRJ); ${ }^{4}$ Laboratory of Tumor Immunology, Leopoldo de Meis Medical Biochemistry Institute (IBqM-UFRJ), Rio de Janeiro, RJ, Brazil; ${ }^{5}$ Division of Cancer, Department of Surgery and Cancer, Imperial College London,

Hammersmith Hospital Campus, London, UK
\end{abstract}

Received May 31, 2014; Accepted July 17, 2014

DOI: $10.3892 /$ ijo.2014.2615

\begin{abstract}
Acute myeloid leukemia (AML) patients' outcome is usually poor, mainly because of drug resistance phenotype. The identification of new drugs able to overcome mechanisms of chemoresistance is essential. The pterocarpanquinone LQB-118 compound has been shown to have a potent cytotoxic activity in myeloid leukemia cell lines and patient cells. Our aim was to investigate if LQB-118 is able to target FoxO3a and FoxM1 signaling pathways while sensitizing AML cell lines. LQB-118 induced apoptosis in both AML cell lines HL60 (M3 FAB subtype) and U937 (M4/M5 FAB subtype). Cell death occurred independently of alterations in cell cycle distribution. In vivo administration revealed that LQB-118 was not cytotoxic to normal bone marrow-derived cells isolated from mice. LQB-118 induced FoxO3a nuclear translocation and upregulation of its direct transcriptional target Bim, in HL60 cells. However, LQB-118 induced FoxO3a nuclear exclusion, followed by Bim downregulation, in U937 cells. Concomitantly, LQB-118 exposure reduced FoxM1 and Survivin expression in U937 cells, but this effect was more subtle in HL60 cells. Taken together, our data suggest that LQB-118 has a selective and potent antitumor activity against AML cells with distinct
\end{abstract}

Correspondence to: Dr Raquel C. Maia, Laboratório de HematoOncologia Celular e Molecular, Programa de Hemato-Oncologia Molecular, Instituto Nacional de Câncer (INCA), Praça da Cruz Vermelha, 23, 6 andar, Rio de Janeiro, RJ 20230-130, Brazil

E-mail: rcmaia@inca.gov.br

*Contributed equally

Key words: acute myeloid leukemia, apoptosis, FoxO3a, FoxM1, pterocarpanquinone LQB-118 compound molecular subtypes, and it involves differential modulation of the signaling pathways associated with FoxO3a and FoxM1 transcription factors.

\section{Introduction}

Acute myeloid leukemia (AML) is a clonal hematopoietic disorder characterized by multiple genetic abnormalities in normal hematopoietic stem cells, which results in accumulation of immature cells - the blasts (1). AML is a highly heterogeneous disease and is categorized into eight FAB (French-American-British) subtypes, based on morphological, cytochemical, and immunophenotypic characteristics of leukemic blasts (2). In addition, genetic and molecular alterations can stratify specific groups and determine their prognosis, since some entities exhibit quite different clinical outcomes (3). AML treatment is mainly based on anthracyclines and cytarabine (4) and has changed little in the past three decades, except for the acute promyelocytic leukemia subtype, in which combination therapy with both anthracycline and transretinoic acid has resulted in a high complete remission rate (5). However, for the other AML subtypes, complete remission rates seldom exceed $70 \%$ in younger patients and $50 \%$ in older patients (4). For adults, 5-year overall survival is between 30 and $50 \%$, with even more disappointing results in terms of disease-free survival, as most patients relapse within 3-5 years from diagnosis $(6,7)$. Taking into account that AML patients have a poor clinical outcome, which is mainly associated with chemoresistance or relapse after previous response (8), and that there has not been any significant advance in treatment it is crucial to develop innovative therapeutic approaches to increase response rates and survival for AML patients.

Pterocarpanquinones are hybrid molecules originated from the molecular hybridization of pterocarpans and naphtoquinones (9), which have been shown to have antitumoral activities 
$(9,10)$. Among them,LQB-118 emerged as one of the most promising compound, because it was able to induce high apoptotic indexes in cells from chronic myeloid leukemia (CML) patients with the multidrug resistance (MDR) phenotype (11), while displaying little toxicity toward peripheral blood mononuclear cells (PBMC) derived from healthy individuals (12). This effect involves not only changes in mitochondrial membrane potential and regulation of the endoplasmic reticulum stress pathway (13), but also inhibition of both the Survivin and XIAP antiapoptotic proteins and the drug efflux transporter P-glycoprotein (Pgp) (11), proteins known to impact prognosis and confer resistance to chemotherapy in multiple leukemia models, including myeloid leukemia (14-20). In AML cells, we have described that the LQB-118 compound triggers apoptosis and suppresses Survivin and XIAP expression in cells from AML patients displaying variable levels of Pgp activity and expression (21). Moreover, there was no nuclear translocation of the NF- $\mathrm{KB}$ oncogenic transcription factor, differently from the effect observed after treatment of the AML Kasumi cell line with idarubicin, an anthracyclin commonly used in AML treatment (21). Nevertheless, the mechanisms of action of the LQB-118 compound and pathways involved in its antitumoral effect in AML cells are not fully elucidated.

Forkhead box (Fox) transcription factors are crucial regulators of a wide range of key biological processes and some are also deregulated in cancer (22). FoxO3a is a member of the Fox class ' $\mathrm{O}$ ' subfamily frequently found in AML leukemic blasts (23). It is known to orchestrate signaling pathways involved in apoptosis, cell cycle arrest, metabolism, DNA repair and response to stress, through modulating transcription of direct targets, such as Bim, p27 $7^{k i p l}$, glucose-6-phosphatase, GADD45a, and manganese superoxide dismutase ( $\mathrm{MnSod})$, respectively $(24,25)$. Conversely, the Fox protein M1 (FoxM1) acts like a typical oncogene and transcriptionally activates genes associated with cell cycle progression (i.e., cyclin B1, Aurora-B kinase, centromere protein A and B, cdc25, and Survivin) $(26,27)$. In AML, aberrant FoxM1 expression was found to contribute to proliferation of leukemic blasts (28). The modulation of FoxO3a and FoxM1 transcription factors has been shown to mediate the cytotoxic and cytostatic effects of multiple chemotherapeutic agents, playing a pivotal role in determining drug sensitivity and resistance (29).

In this study, we investigated the ability of the LQB-118 compound to sensitize AML cell lines and target FoxO3a and FoxM1 transcription factors. Our data show that LQB-118 can induce high apoptotic indexes in AML cells of distinct molecular subtypes. LQB-118 promotes FoxO3a nuclear translocation and Bim upregulation in HL60 cells. However, FoxO3a is relocated to the cytoplasm and Bim expression is suppressed in U937 cells, in which chemosensitivity correlates with FoxM1 and Survivin inhibition, suggesting that the LQB-118 compound is able to differentially target Fox transcription factors to sensitize AML cells of distinct molecular subtypes. Studying different AML molecular subtypes is important for preclinical drug development, as they display different outcomes and drug resistance patterns in clinical scenario. In addition, LQB-118 was administered to Swiss mice without detectable toxicity to normal bone marrow-derived cells, which indicates that it has selectivity for tumor cells.

\section{Materials and methods}

Materials. The pterocarpanquinone LQB-118 was synthesized in the Laboratory of Bioorganic Chemistry, at the Federal University of Rio de Janeiro (UFRJ) as described (10). The compound was dissolved in dimethyl sulfoxide (DMSO), and serial dilutions in culture medium were performed before use. DMSO in the concentration paired to the highest concentration of the LQB-118 compound was used as a vector control in all experiments. The LQB-118 compound was used at concentrations of 1.5, 3, 6 and $9 \mu \mathrm{M}$, which were demonstrated to cause no toxicity to PBMC isolated from healthy individuals (12). All materials are described in detail in each section.

Animals. Swiss mice were bred in the animal facilities at the Leopoldo de Meis Biochemistry Institute from UFRJ (IBqM-UFRJ), and housed under standard laboratory conditions $\left(20-25^{\circ} \mathrm{C}, 12\right.$-h light regimen) with free access to water and standard chow (ad libitum). Two-month female mice were used in the experiments. Procedures were approved by the Center of Health Sciences Ethics Committee for Animal Use (CEUA-CCS, UFRJ) under the protocol IBQM082.

Isolation of bone marrow cells. Mice were anaesthetized with ethyl ether (Reagen, Rio de Janeiro, Brazil) and euthanized by cervical dislocation. Bone marrow was aspirated from femoral bones with a syringe containing RPMI-1640 medium (Sigma-Aldrich Corp. St. Louis, MO, USA). Bone marrow cells were homogenized in cold RPMI-1640 medium, centrifuged at $200 \mathrm{~g}$ for $10 \mathrm{~min}$ and then resuspended in $3 \mathrm{ml}$ of cold RPMI medium supplemented with $5 \%$ fetal bovine serum (FBS; Gibco, Grand Island, NY, USA). The cell suspension was incubated with $0.08 \%$ Trypan blue staining (Sigma-Aldrich) and counted in an optical microscope.

$L Q B-118$ in vivo administration. The in vivo concentration of LQB-118 used was based upon its in vitro $\mathrm{EC}_{50}$ obtained from previous work. Calculations were made considering a medium of $30 \mathrm{~g}$ weight and $4 \mathrm{ml}$ of bodily fluids, the final concentration of $3.8 \mathrm{mg} / \mathrm{kg}$ is representative of $\sim 15 \mathrm{X} \mathrm{EC} \mathrm{E}_{50}$. Mice were intraperitoneally injected with $3.8 \mathrm{mg} / \mathrm{kg}$ LQB-118 diluted in phosphate buffered saline (PBS; Sigma-Aldrich) and DMSO (Sigma-Aldrich). Control group received only the vehicle (diluent).

Ex vivo analysis of bone marrow subpopulations. Cells from the bone marrow of femoral bones were washed with PBS supplemented with 5\% FBS (Gibco), resuspended in $300 \mu \mathrm{l}$ of RPMI-1640 medium for the final concentration of $10^{6}$ cells $/ \mathrm{ml}$. Subsequently, cells were analyzed by flow cytometry in a FACSCalibur (Becton-Dickinson, San Jose, CA, USA). Ten thousand cells were acquired based on forward and side scatter parameters, representative of cell size and granulosity. Analysis was performed on Summit v4.3 software (Dako Colorado, Inc., Fort Collins, CO, USA).

Cell lines. The human AML-derived cell lines HL60 and U937 were obtained originally from American Type Culture Collection (ATCC; VA, USA) and cultured in RPMI-1640 medium (Gibco; BRL, UK) supplemented with $10 \%$ heat- 
a

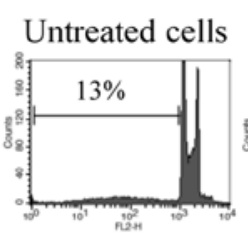

b

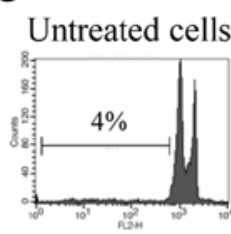

C
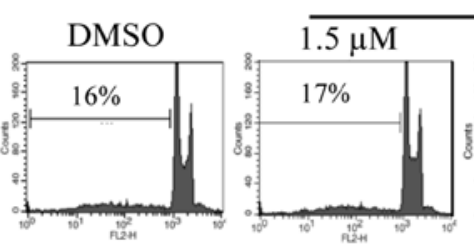

HL60

LQB-118

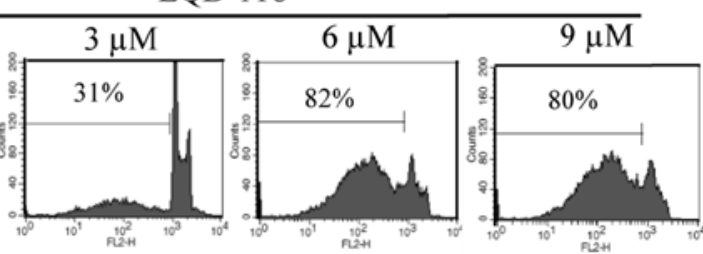

U937
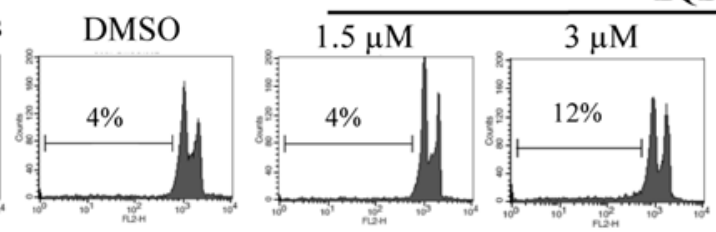

LQB-118

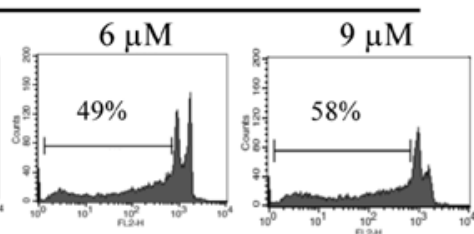

HL60

LQB-118
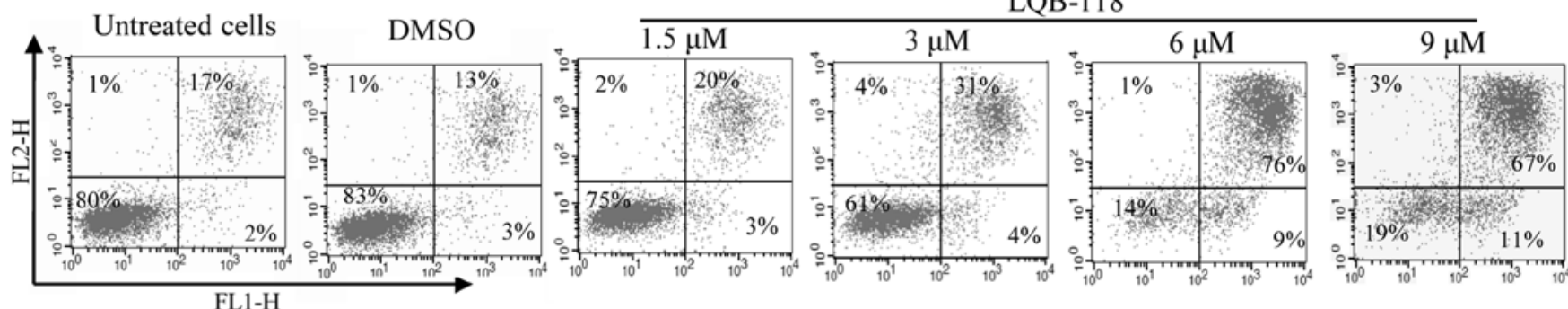

d

U937

LQB-118
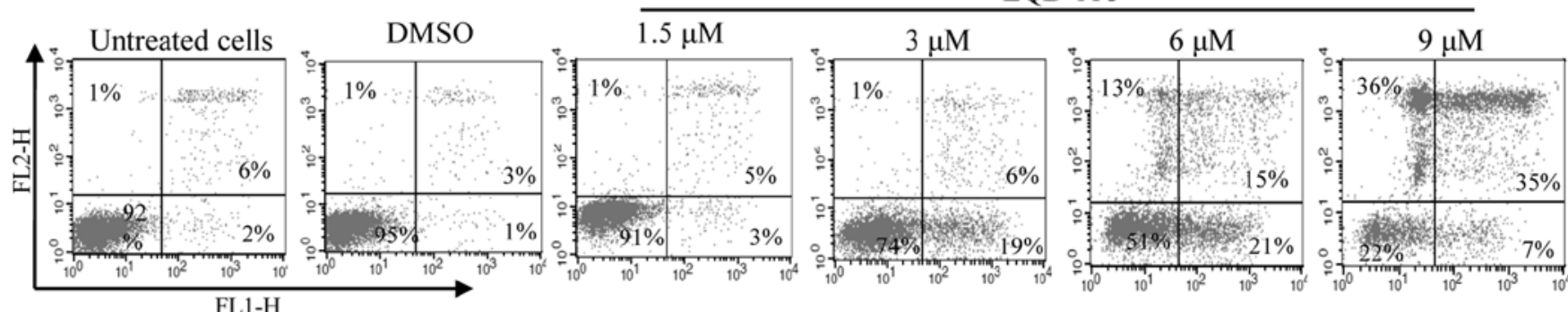

Figure 1. Flow cytometry analysis of LQB-118-induced cell death in HL60 and U937 AML cell lines. HL60 and U937 cell lines were exposed to 1.5, 3, 6 and $9 \mu \mathrm{M}$ of LQB-118 and to dimethyl sulfoxide (DMSO) and collected after a 24-h exposure. (a and b) DNA fragmentation was evaluated in the cell lines and estimated by the percentage of cells in the sub-G0/G1 phase. (c and d) Cells were stained with Annexin V and propidium iodide for detection of Annexin V positivity. DMSO was used as a vector control for the highest concentration. The images are representative of three independent experiments.

inactivated FBS and glutamine $(2 \mathrm{mM})$ and maintained in a humidified atmosphere at $37^{\circ} \mathrm{C}$ and $5 \% \mathrm{CO}_{2}$. HL60 cells are derived from promyelocytic leukemia (M3 FAB subtype) $(30,31)$ and U937 cells, from monocytic type (M4/M5 subtypes) (32). The cell lines were genotyped for confirmation of authenticity by the Sonda Laboratory at UFRJ and monitored for mycoplasm contamination. Cells were exposed to LQB-118 for $24 \mathrm{~h}$ at a density of $2 \times 10^{5} / \mathrm{ml}$.

Assessment of DNA content. After LQB-118 treatment, $2 \times 10^{6}$ cells were washed twice in PBS and incubated with $500 \mu \mathrm{l}$ of propidium iodide (PI) staining solution (PI $50 \mu \mathrm{g} /$ $\mathrm{ml}$ diluted in citrate buffer $4 \mathrm{mM}$ and Triton X-100 0.3\%) and $500 \mu \mathrm{l}$ ribonuclease A (RNAse $100 \mu \mathrm{g} / \mathrm{ml}$ diluted in citrate buffer $40 \mathrm{mM}$ ) for $15 \mathrm{~min}$ at room temperature. DNA content was determined on a flow cytometer (FACSCalibur; BD Biosciences, San Jose, CA, USA) and a total of ten thousand events were acquired per sample. Analysis was performed using CellQuest Pro software. This assay was performed to analyze the cell cycle profile and assess DNA fragmentation, which was quantified by the percentage of cells in sub-G0'G1 phase.

Apoptosis assay. For detection of apoptosis, the Annexin V assay (Genzyme Diagnostics, Cambridge, MA, USA) was performed. Cells were stained with FITC-labeled Annexin V as previously described (11). The compound-induced apoptotic index was analyzed using flow cytometry, and results were calculated as a percentage of Annexin V-positive cells in the presence of the drug subtracting the positivity in the absence of the drug.

Detection of caspase-3 expression using flow cytometry. After exposure to LQB-118 for $24 \mathrm{~h}$, a total of $2 \times 10^{6}$ cells were washed with PBS and centrifuged. Then, cells were submitted to a caspase-3 staining protocol as previously described (20). Anti-active caspase-3 antibody was purchased from BD Biosciences. For each condition, a negative control without 
a
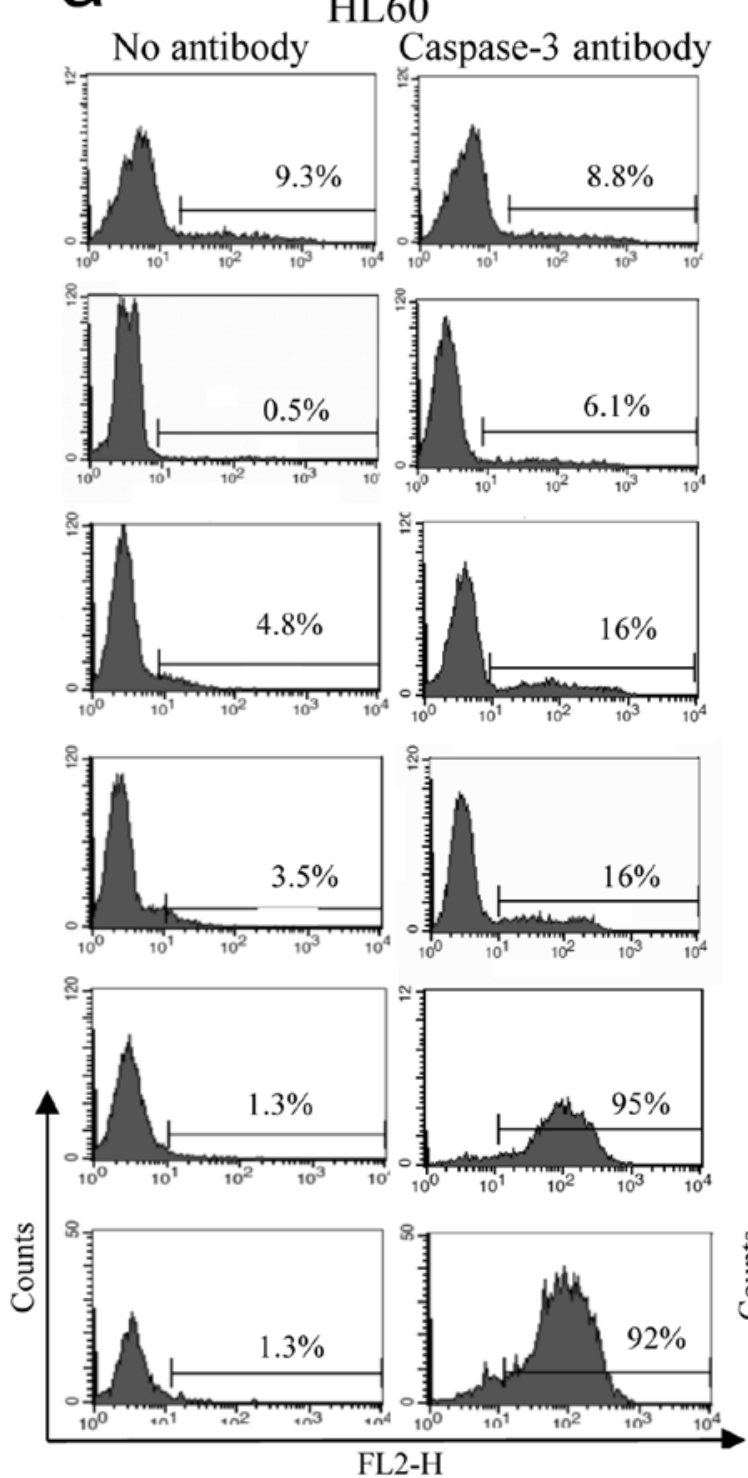
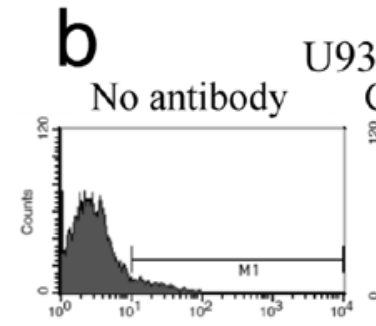

Caspase-3 antibody
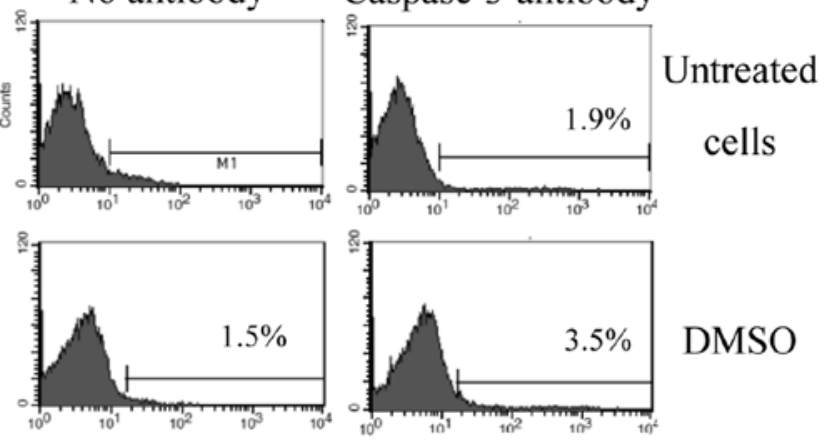

DMSO
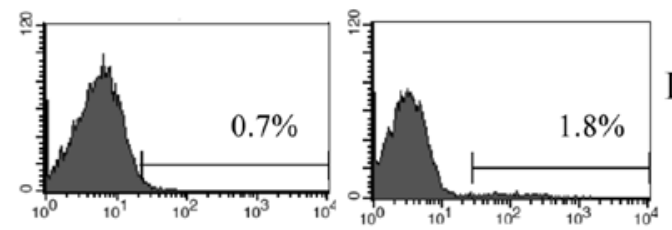

LQB-118

$1.5 \mu \mathrm{M}$
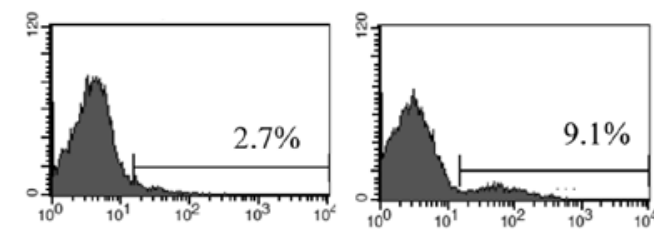

LQB-118

$3 \mu \mathrm{M}$
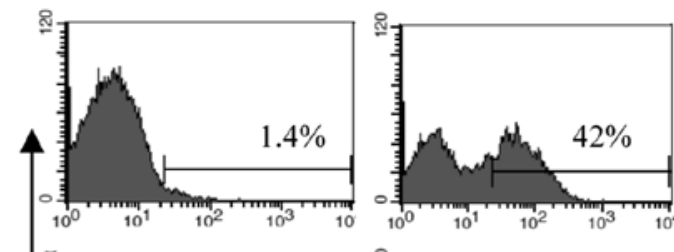

LQB-118

$6 \mu \mathrm{M}$

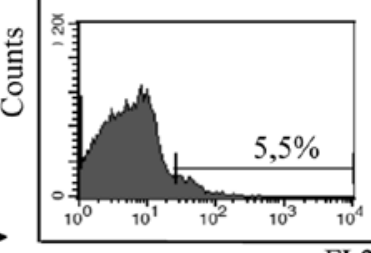

FL2-H

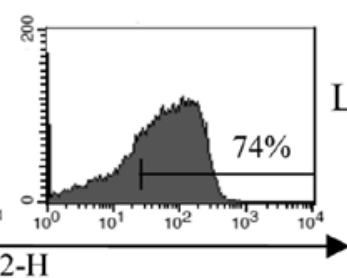

LQB-118

$9 \mu \mathrm{M}$

Figure 2. Profile of caspase-3 activation mediated by the LQB-118 compound in HL60 and U937 cells. HL60 (a) and U937 (b) cell lines were incubated with $1.5,3,6$ and $9 \mu \mathrm{M}$ of LQB-118 and with dimethyl sulfoxide (DMSO). After $24 \mathrm{~h}$ of incubation, cells were harvested and analyzed for caspase-3 activation using flow cytometry as described in Materials and methods. The percentages of LQB-118-induced caspase-3 activation were calculated by subtracting the values in the caspase- 3 stained from the negative control samples (no antibody). The histograms are representative of three independent experiments.

antibody was also analyzed. The results were expressed as the percentage of cell with caspase-3 positivity.

Immunofluorescence. After LQB-118 treatment for $24 \mathrm{~h}$, an immunofluorescence assay using an anti-FoxO3a monoclonal antibody was performed. Cytospins were prepared with HL60 and U937 $10^{5}$ cells treated or not with $1.5 \mu \mathrm{M}$ of LQB118 and DMSO. Cytospins were fixed with $4 \%$ paraformaldehyde for 20 min, and permeabilized with 1\% PBS (pH 8.0)-Triton X-100 for $10 \mathrm{~min}$. Cells were blocked with PBS (pH 8.0) containing $3 \%$ BSA for $1 \mathrm{~h}$ and then, stained with a monoclonal antibody against FoxO3a (1:200 dilution, Cell Signaling, Danvers, MA, USA) diluted in PBS ( $\mathrm{pH}$ 8.0) 1\% bovine serum albumin (BSA) overnight. The antibody-antigen complexes were detected by incubation for $1 \mathrm{~h}$ with secondary anti-rabbit Alexa Fluor 488 or 594 antibodies (1:300 dilution, Life Technologies, NY, USA) in PBS (pH 8.0) 1\% BSA. Cells were washed and treated with
DAPI (1:1,000, 4',6-diamidino-2-phenylindole) for $10 \mathrm{~min}$ for nuclear staining. Coverslips were mounted with $\mathrm{N}$-propylgallate and the cells were subsequently analyzed with Nikon fluorescence microscope using the Nikon software (Leica Microsystem).

Western blot analysis. For detection of Bim, FoxM1, Survivin, Beclin 1 and $\beta$-actin expression, pellets of $2 \times 10^{6}$ were harvested and submitted to protein extraction and immunoblotting as previously described (17). Primary antibodies comprised Bim (1:1,000 dilution; Stressgen, Brussels, Belgium), FoxM1 (1:500 dilution; Cell Signaling), Survivin (1:1,000 dilution; R\&D Systems, Minneapolis, MN, USA), Beclin1 (1:1,000; BD Biosciences), and $\beta$-actin (1:5,000 dilution; Sigma-Aldrich). Secondary anti-mouse (1:500 dilution) and anti-rabbit (1:1,000 dilution) were purchased from GE Healthcare (Buckinghamshire, UK). Antibody complexes were visualized 
a

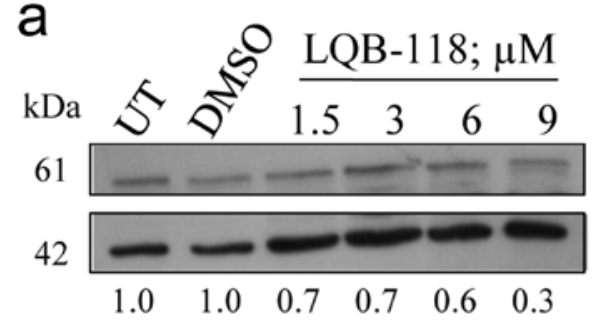

C

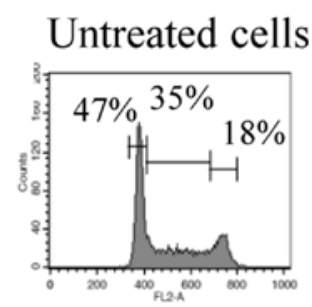

HL60
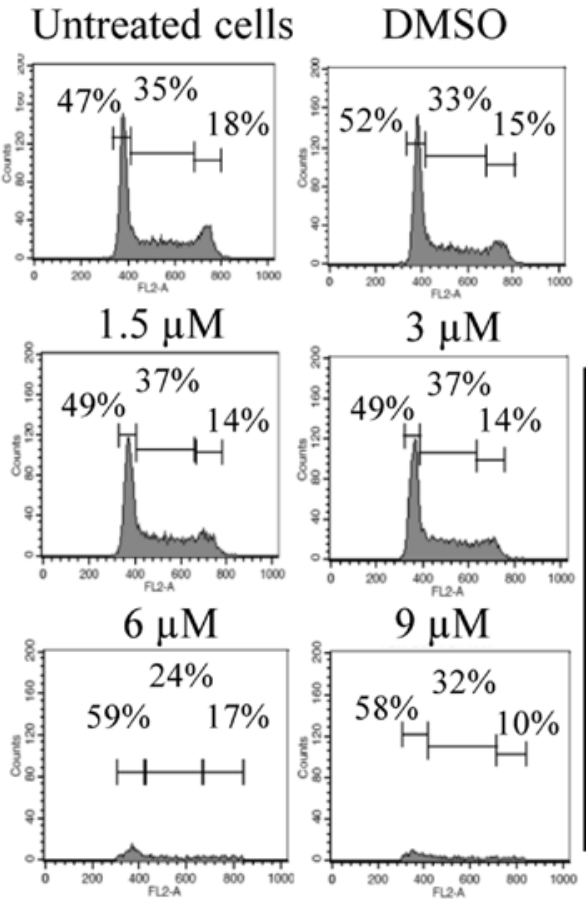

b

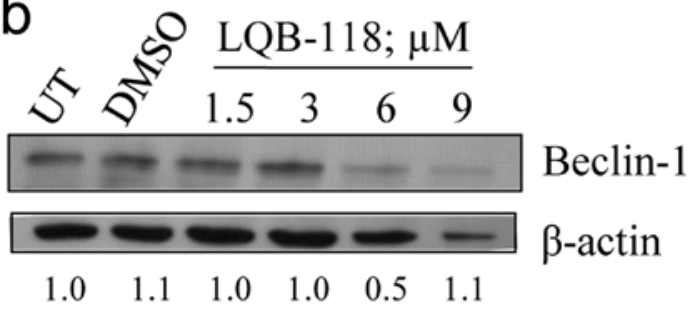

d

\section{U937}

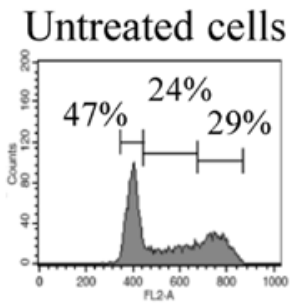

\section{DMSO}
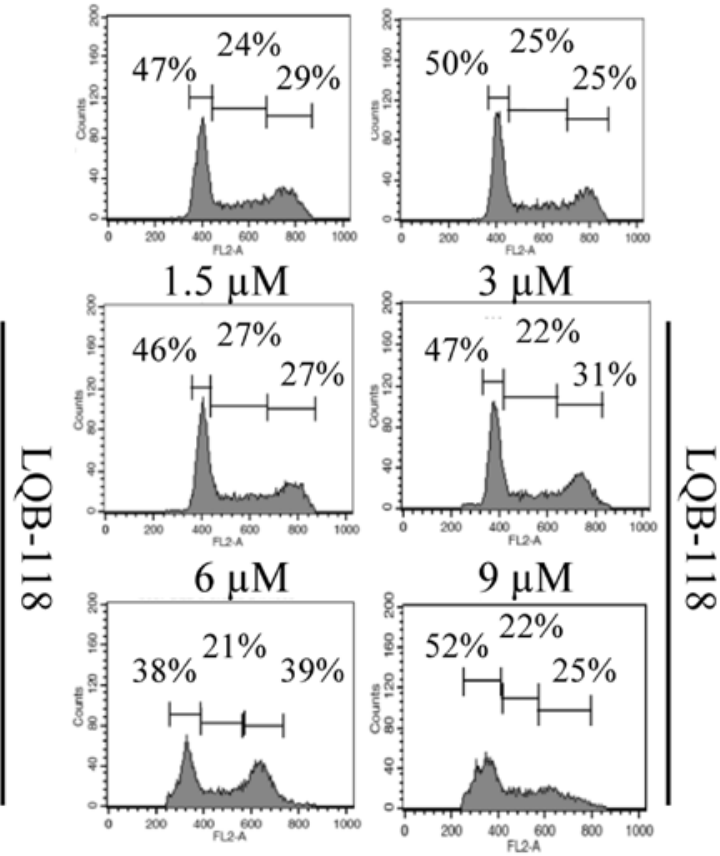

Figure 3. Analysis of Beclin1-mediated autophagy and cell cycle profile changes induced by the LQB-118 compound in HL60 and U937 cells. The cell lines were exposed to 1.5, 3, 6 and $9 \mu \mathrm{M}$ of LQB-118 and to dimethyl sulfoxide (DMSO) and collected after a 24-h exposure. (a and b) Cells were lysed for western blotting. The values below the blots indicate the densitometric ratio between the protein and $\beta$-actin. (c and d) DNA content was evaluated using cells stained with propidium iodide and analyzed using flow cytometry. UT, untreated cells. The graphs are representative of three independent experiments.

using the ECL detection system (GE Healthcare) and band intensities were quantified using the VisionWorks software. Protein expression was normalized with respect to $\beta$-actin.

Statistical analysis. Statistical analysis was performed using GraphPad Prism 4.0 software (San Diego, CA, USA). Statistical significance was calculated by non-parametric and unpaired Student's t-test since experiments compared different groups of animals. Data presented normal distribution according to normality test.

\section{Results}

The LQB-118 compound induces apoptosis in AML cell lines derived from different $F A B$ subtypes. Recently, we demonstrated that the novel compound LQB-118 is capable of sensitizing the Kasumi AML cell line, derived from the M2 FAB subtype, to undergo apoptosis (21). Since AML is a highly heterogeneous myeloproliferative disease $(1,33)$, the first step of this study was to investigate if LQB-118 cytotoxic effects extend to cell lines derived from other AML FAB subtypes. As observed in Fig. 1a and b, exposure to LQB-118 compound for $24 \mathrm{~h}$ resulted in high percentages of DNA fragmentation, mainly at concentrations of 6 and $9 \mu \mathrm{M}$. We also found that both cell lines had an increase in Annexin V staining after LQB-118 treatment in a dose-dependent manner (Fig. 1c and d), suggesting that the compound might exert its effects on AML cells by triggering apoptosis. To confirm these data, we assessed caspase-3 levels and observed that LQB-118 induced caspase-3 activation in both cell lines (Fig. 2). It is important to highlight that HL60 cells were slightly more sensitive than U937 cells in all cytotoxicity assays performed. We also questioned if the LQB-118 compound would somehow modulate the autophagic process. Our results show that the levels of Beclin1, which is an important regulator of autophagy $(34,35)$, were not induced upon LQB-118 exposure (Fig. 3a and b). This suggests that the compound does not induce Beclin1-mediated autophagy. Our data show that the LQB-118 compound is highly effective in inducing apoptotic cell death in AML cell lines derived from different FAB subtypes.

LQB-118-induced apoptosis occurs independently of changes in the cell cycle. Since many chemotherapeutic agents exert their cytotoxic effects through the modulation of the phases of 

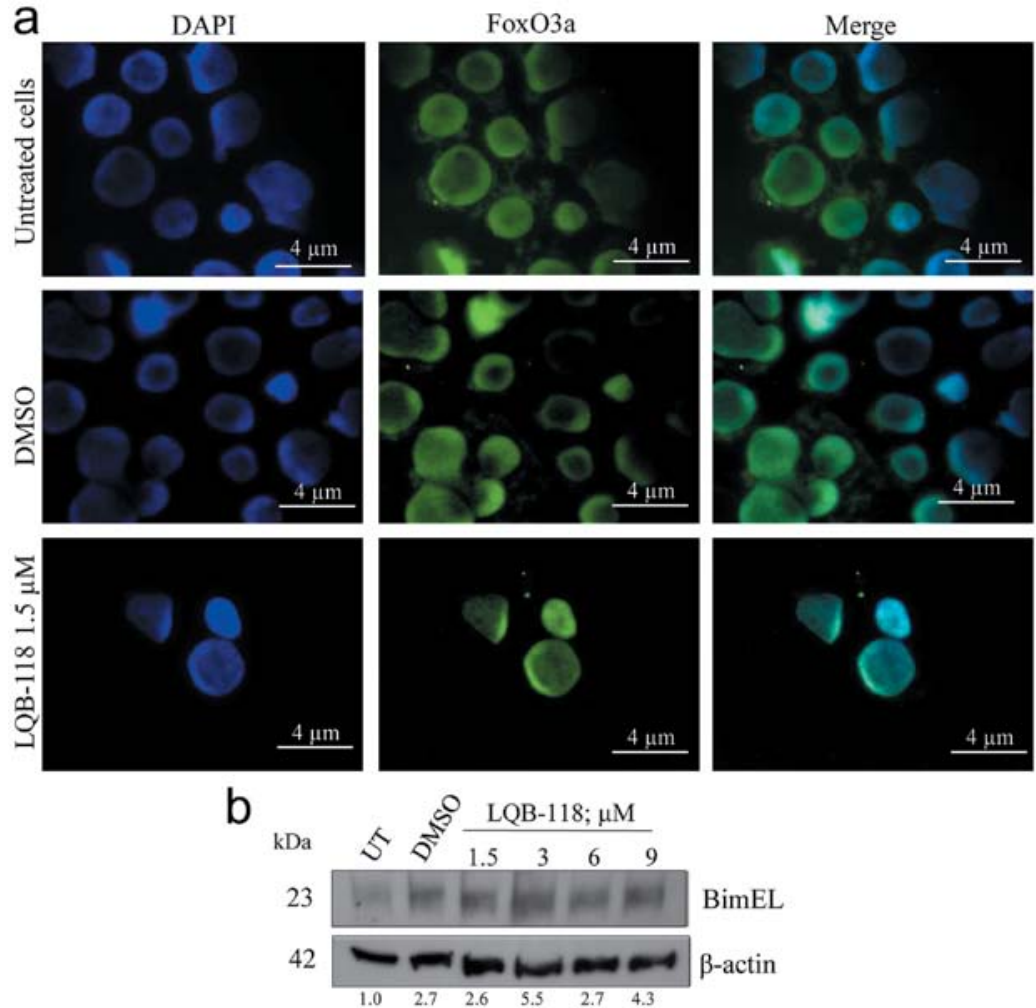

Figure 4. Modulation of FoxO3a subcellular localization and Bim expression after treatment of HL60 cells with the LQB-118 compound. (a) HL60 cells were exposed to 1.5 of LQB-118 and to dimethyl sulfoxide (DMSO) and harvested after $24 \mathrm{~h}$. Then, cells were stained with anti-FoxO3a antibody (green) and DAPI (blue) and analyzed using immunofluorescence. Scale bars, $4 \mu \mathrm{M}$. (b) Cells were treated with 1.5, 3, 6 and $9 \mu \mathrm{M}$ of LQB-118 and with DMSO and lysed for western blotting. The values below the blots indicate the densitometric ratio between the protein and $\beta$-actin. Bim indicates Bim-XL. UT, untreated cells. The images are representative of three independent experiments.

a
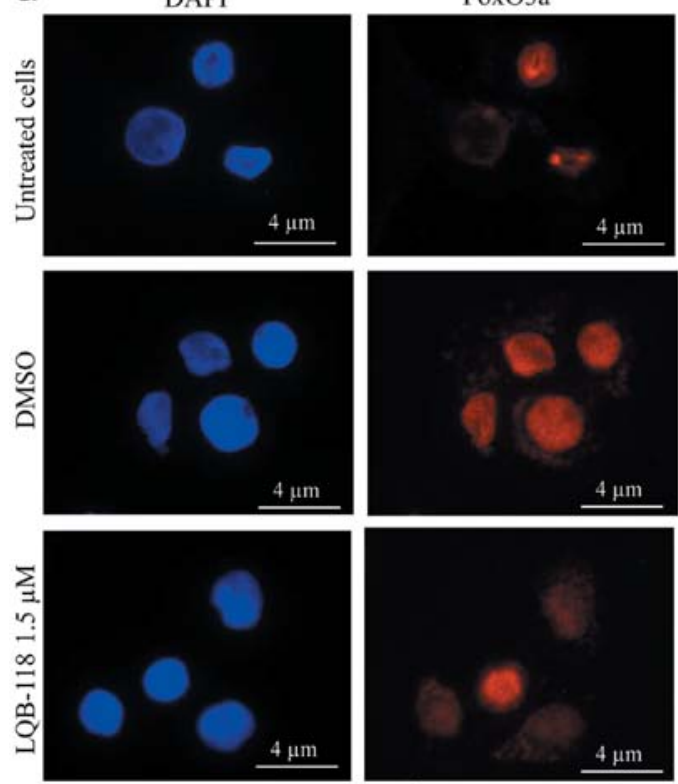

b

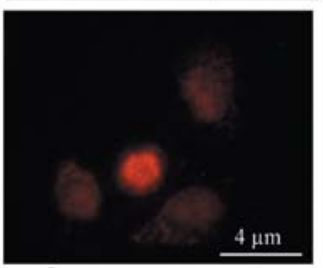

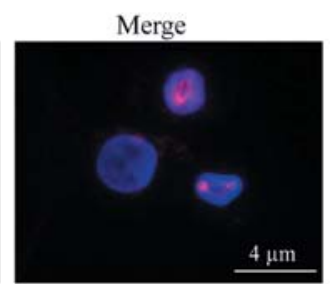
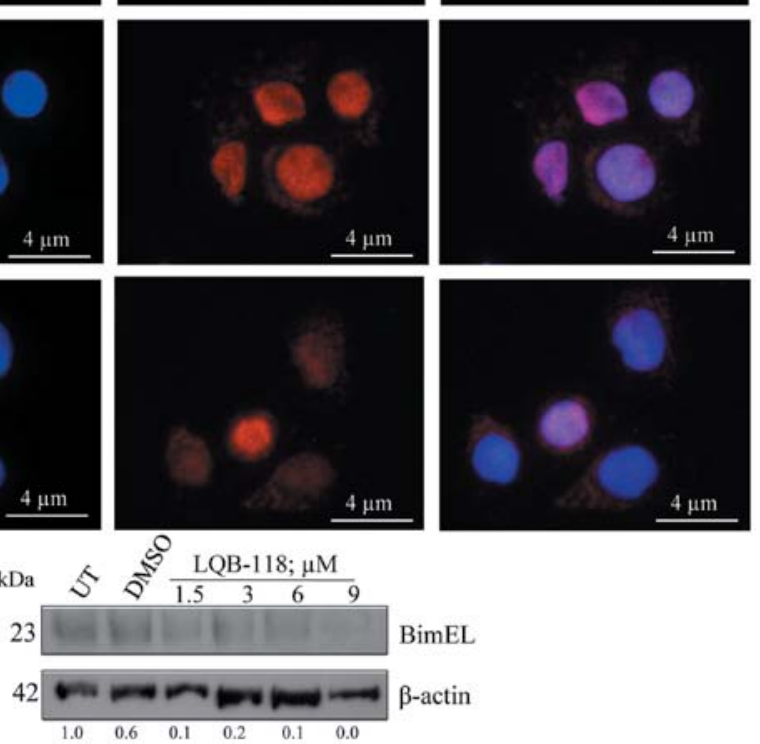

Figure 5. Modulation of FoxO3a subcellular localization and Bim expression after treatment of U937 cells with the LQB-118 compound. (a) U937 cells were exposed to 1.5 of LQB-118 and to dimethyl sulfoxide (DMSO) and harvested after $24 \mathrm{~h}$. Then, cells were stained with anti-FoxO3a antibody (green) and DAPI (blue) and analyzed using immunofluorescence. Scale bars, $4 \mu \mathrm{M}$. (b) Cells were treated with 1.5, 3, 6 and $9 \mu \mathrm{M}$ of LQB-118 and with DMSO and lysed for western blotting. The values below the blots indicate the densitometric ratio between the protein and $\beta$-actin. Bim indicates Bim-XL. UT, untreated cells. The images are representative of three independent experiments. 
a
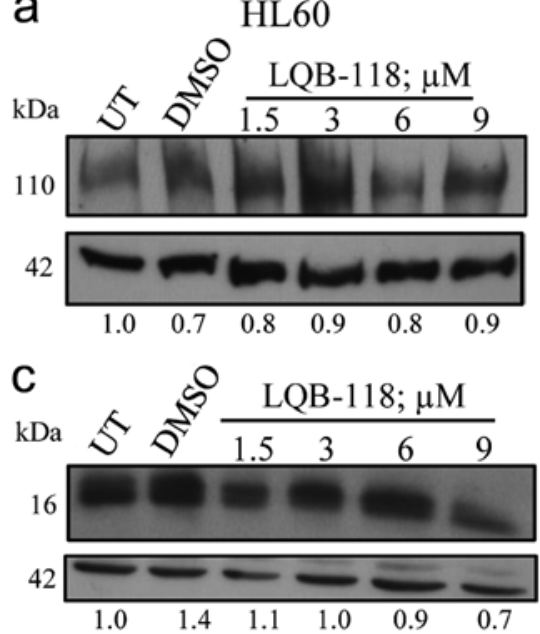

b

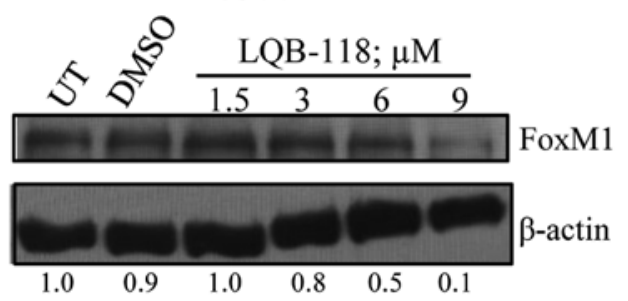

d

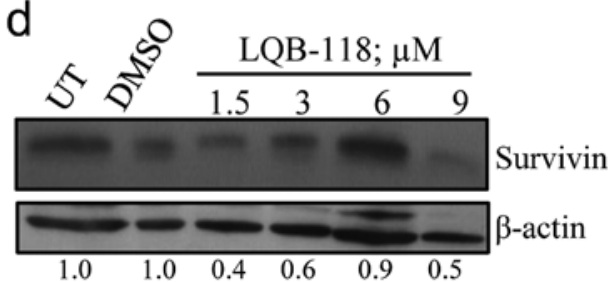

Figure 6. Western blot analysis of LQB-118-mediated modulation of FoxM1 and Survivin expression. HL60 and U937 cells were treated with 1.5, 3, 6 and $9 \mu$ M of LQB-118 and with DMSO for $24 \mathrm{~h}$. Then, cell lines were lysed for western blotting and had FoxM1 (a and b) and Survivin (c and d) expression analyzed. The values below the blots indicate the densitometric ratio between the protein and $\beta$-actin. UT, untreated cells. The images are representative of three independent experiments.

cell cycle, we investigate the ability of LQB-118 compound to interfere with the cell cycle. The histograms shown in Fig. 3c and $3 \mathrm{~d}$ indicate that the treatment of both cell lines with the compound did not result in significant changes in cell cycle distribution. This indicates that apoptosis induced by LQB-118 occurs independently of alterations in the cell cycle profile.

LQB-118 treatment results in FoxO3a transcription factor relocation and Bim induction in HL60 cells, but not in U937 cells. Next, we investigated if LQB-118 compound would be able to target FoxO3a, as it is known that the transcription factor can mediate the cytotoxic effects of several drugs in cancer cells (24). We could observe that both cell lines displayed a diffuse, predominantly nuclear pattern of FoxO3a staining (Fig. 4a and 5a). Upon LQB-118 treatment at $1.5 \mu \mathrm{M}$, we observed nuclear translocation of FoxO3a in HL60 cells (Fig. 4a). We also analyzed Bim expression, which is a direct transcriptional target for FoxO3a, and LQB-118 was able to induce Bim expression (Fig. 4b) (36). Interestingly, LQB-118 induced nuclear exclusion of FoxO3a in U937 cells (Fig. 5a). Corroborating these data, there was no Bim upregulation, and its expression was inhibited in a dose-dependent manner (Fig. 5b). It is relevant to emphasize that U937 cells displayed a resistant-phenotype at lower concentrations (e.g., $1.5 \mu \mathrm{M}$ ). Notably, immunofluorescence experiments were not performed after treatment with 3, 6 and $9 \mu \mathrm{M}$ of LQB-118 because cells were not suitable for morphologic analysis. Together, these data indicate that LQB-118 can promote a differential modulation of FoxO3a pathway in AML cells with distinct molecular characteristics.

The LQB-118 compound promotes repression of FoxM1 transcription factor in U937 cells. Because FoxM1 has been shown to induce cell proliferation in AML cells (28) its suppression would be a viable approach for these leukemic subtypes. As a consequence, we questioned if FoxM1 would be a target for the LQB-118 compound. Fig. 6b shows that
FoxM1 expression is abolished in U937 cells upon treatment with $9 \mu \mathrm{M}$ of LQB-118. This effect was not so pronounced in HL60 cells, in which FoxM1 levels remained almost unaltered (Fig. 6a). Survivin expression was evaluated because it can be positively regulated by FoxM1 and it has a known role in drug resistance and as a therapeutic target $(28,37)$. After LQB-118 treatment, we observed that U937 cells displayed a decrease in Survivin levels, which was less pronounced in HL60 cells (Fig. 6c and d). These data show that the LQB-118 compound targets the FoxM1 pathway to induce apoptosis in U937, but not in HL60 cells.

$L Q B-118$ compound is not cytotoxic to normal bone-marrow derived cells. Next, we asked if the LQB-118 compound would have a further cytotoxic effect on normal bone marrow cells. To investigate this conjecture, we intraperitoneally injected healthy Swiss mice with LQB-118, and collected cells from their bone marrow. As shown in Fig. 7a, there was no statistical significance between the absolute cell count from LQB-118treated mice and those injected with the diluents. We also analyzed bone marrow cellular populations and found that the LQB-118 compound did not alter the distribution of the subpopulations when compared to control group (Fig. 7b and c). These results indicate that LQB-118 has a selective antitumoral activity, since it does not affect normal cells from the bone marrow.

\section{Discussion}

AML is a highly heterogeneous disease, characterized by a diverse spectrum of genetic abnormalities and the accumulation of blastic cells $(1,2)$. Despite effort having been made, no significant advances were observed in AML treatment, complete remission, and survival rates, except for the APL subtype $(4,5)$. Statistics from the Surveillance Epidemiology and End Results (SEER) show that the overall 5-year relative survival is $24.2 \%$, among AML population in general. Poor outcomes 
a

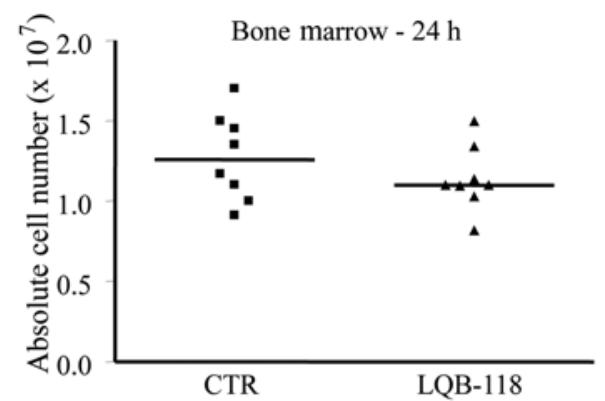

b

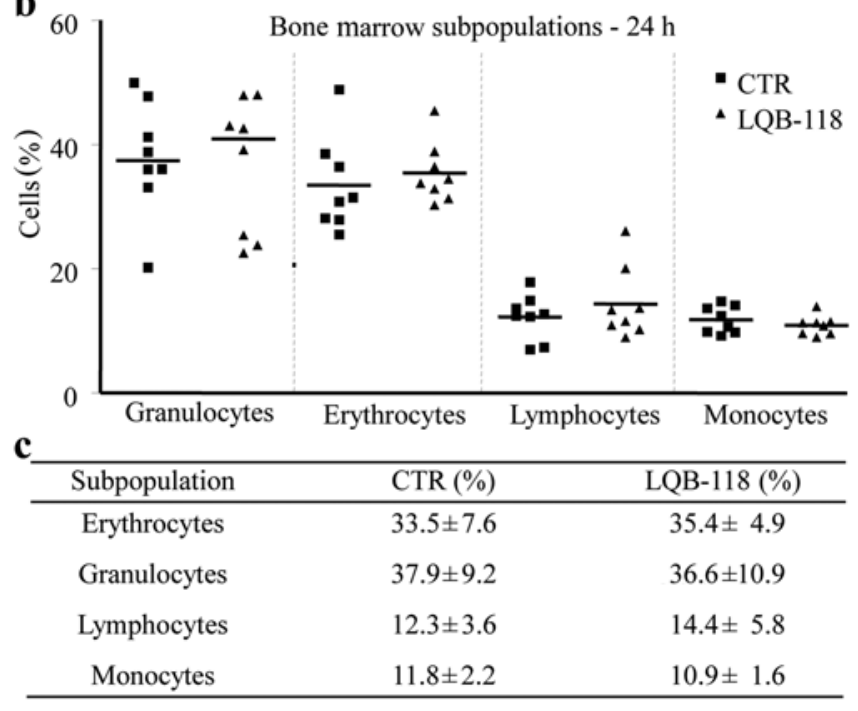

Figure 7. Effect on bone marrow cells after LQB-118 administration. (a) Swiss mice were intraperitoneally injected with $3.8 \mathrm{mg} / \mathrm{kg} \mathrm{LQB}-118$. After $24 \mathrm{~h}$ animals were euthanized and cells from bone marrow were obtained by bone marrow aspiration, incubated with Trypan blue dye, and only viable cells were counted on optical microscope. (b) Cells were collected the same way, and then analyzed using flow cytometry. Percentages representative of each cell subpopulation were based on cell size and granulosity. CTR, animals injected with diluent. No statistical difference was found on each cell subtype $(\mathrm{p}>0.05)$. Lines at the median of each cell population, $\mathrm{n}=8$. (c) Distribution of the bone marrow cell subpopulations of Swiss mice. Results reported as percentage of each cell subpopulation $\pm \mathrm{SD}, \mathrm{n}=8$.

in AML patients are closely related to chemoresistance and/ or disease relapse (8). Considering that $\sim 15,000$ people were diagnosed with AML in the USA in 2013 (38), there is an urgent need for research on treatment and development of novel therapeutic strategies. AML cell lines represent in vitro models for studying preclinical drug development. In recent studies, the novel pterocarpanquinone LQB-118 demonstrated a satisfactory cytotoxic effect against diverse neoplastic cell lines, with little toxicity towards $\operatorname{PBMC}(10,12)$, indicating that it might be selective in killing tumor cells. Interestingly, the LQB-118 compound induced apoptosis in cells from AML and CML patients regardless of the MDR phenotype $(11,21)$, which strongly suggests that it may bypass mechanisms of resistance while sensitizing tumor cells. Consistent with this, LQB-118 inhibited the expression of Survivin and XIAP antiapoptotic proteins and Pgp efflux transporter in AML and CML cell lines $(11,21)$ and also triggered cascades involving the endoplasmic reticulum stress pathway and depolarization of mitochondrial membrane potential (13). Although these previous studies provided some insights into LQB-118 mechanisms of action, they also prompted us to investigate whether its effect extends to other AML subtypes and which signaling pathways are regulated during chemosensitivity.

In the present study, we evaluated the effects of LQB-118 compound in HL60 (M3 subtype) and U937 (M5 subtype) AML cell lines and observed that it induced apoptosisassociated DNA fragmentation in both cell lines. Additionally, there was a great increase in the population of cells positive for Annexin V, when compared with the control of untreated cells. Confirming these data, we found that $\sim 70$ and $90 \%$ of U937 and HL60 cells, respectively, exhibited activated caspase-3 levels when exposed to LQB-118, suggesting that the compound is able to induce high apoptotic indexes in AML cells. Although HL60 cells were shown to be more sensitive to the compound even at the lowest concentrations, high LQB-118 concentrations could trigger U937 cells to undergo apoptosis. The LQB-118-mediated cytotoxic effects previously mentioned occurred independently of changes in cell cycle distribution and had no participation of Beclin1, a critical regulator of the autophagic process $(34,35)$. Reinforcing these data, we have recently demonstrated that LQB-118 displays anticancer activities in the Kasumi cell line (M2 subtype) as well as cells from AML patients (21). Altogether, these results show that the LQB-118 compound is very effective in inducing apoptosis in cells derived from distinct AML molecular subtypes. It is important to emphasize that we observed no toxicity to normal bone marrow cells isolated from LQB-118-treated mice when compared to control. Thus, the low toxicity against these cells is indicative that this pterocarpanquinone has a selective activity against leukemic cells. In 2011, da Cunha-Júnior et al described an anti-leishmanial effect of LQB-118 in BALB/c mice infected with Leishmania amazonensis (39). The compound was administered intraleasionally, orally and intraperitoneally, the latter being effective to control both lesion and parasite growth in a concentration of $4.5 \mathrm{mg} / \mathrm{kg} /$ day, comparable to our study. Furthermore, this concentration did not induce liver damage, since it did not alter serological markers of toxicity such as the enzymes aspartate aminotransferase (AST), alanine aminotransferase (ALT) and creatinine. However, it does not exclude the possibility of the molecule undergoing liver metabolism first before reaching the bone marrow. However, since it selectively induced oxidative stress and apoptosis against L. Amazonensis (40), this possibility seems less likely.

We also explored the signaling pathways regulated by LQB-118 in AML cells. First, we assessed FoxO3a localization, because its transcriptional activity is regulated by nuclear-cytoplasmic shuttling (41) and observed that LQB-118 treatment of HL60 cells resulted in FoxO3a nuclear translocation. Accordingly, the expression of its direct target Bim was upregulated upon LQB-118 exposure. In contrast, LQB-118 treatment of U937 cells led to FoxO3a nuclear exclusion and Bim downregulation. It is important to note that the immunofluorescence was performed with cells exposed to the lowest LQB-118 concentration, which is cytotoxic to HL60, but not to U937 cells. This result suggests that LQB-118 promotes differential modulation of FoxO3a localization in different molecular subtypes and that FoxO3a cytoplasmic localization, and consequent prevention from its transcriptional activity, correlates with LQB-118 resistance in our model. Although some reports propose the idea that FoxO3a might have an oncogenic role in 
AML $(42,43)$ most studies point FoxO3a as a tumor suppressor transcription factor. It was demonstrated that the FLT3 tyrosine kinase promotes phosphorylation-mediated FoxO3a inactivation, repression of $\mathrm{Bim}$ and $\mathrm{p} 27^{\mathrm{kip} 1}$ gene expression and apoptosis inhibition in FLT3-expressing AML cells (44). Also, high phosphorylated FoxO3a levels were found to be an independent predictor of shorter remission duration and drug resistance (44). Corroborating these data, FoxO3a activation has been shown to be an essential event in mediating the effects of transretinoic acid (45), hypomethylating agents (46), Aurora A kinase inhibitors (47) and FLT3 inhibitors (48) in AML cells, through positive regulation of its targets TRAIL, Bim, Puma, and $\mathrm{p} 27^{\mathrm{kip} 1}$. These studies further emphasize FoxO3a usefulness as a target for therapeutic approaches aiming to overcome resistance in AML.

Based on the fact that FoxO3a and FoxM1 transcription factors have opposing functions (49), our next step was to investigate FoxM1 modulation upon LQB-118 treatment. Our results show that FoxM1 expression was progressively decreased in LQB-118-treated U937 cells. Survivin, an antiapoptotic protein known to be transcriptionally regulated by FoxM1, was also downregulated after exposure to the compound. The modulation in the FoxM1/Survivin axis was less pronounced in HL60 cells, mainly at the concentration of $9 \mu \mathrm{M}$, indicating that this signaling pathway undergoes differential modulation by the LQB-118 compound, depending on the molecular subtype. FoxM1 is a master regulator of cell cycle progression (27), whose overexpression was found in a wide variety of tumors (reviewed in ref. 50). Although the role of FoxM1 in AML has not been fully elucidated, FoxM1 was found to be aberrantly expressed in leukemic blast cells $(28,51)$. Consistently, FoxM1 depletion inhibited colony formation in cells derived from AML patients, indicating its relevance for cell cycle progression, and also resulted in Survivin suppression in a panel of cell lines (28). Survivin expression has already been shown to correlate with poor prognosis in AML and chemoresistance, as Survivin knockdown sensitized AML cells to LQB-118 and idarubicin-induced apoptosis $(14,21)$. This group of data suggests that the LQB-118 compound negatively regulates the oncogenic FoxM1/Survivin axis while sensitizing AML cells.

In conclusion, this study demonstrated that the LQB-118 compound can regulate multiple pathways to induce apoptosis and target FoxO3a and FoxM1 transcription factors in AML cells, with no toxicity to normal bone marrow cells. LQB-118 effects were different in cell lines derived from distinct molecular subtypes, which suggests that its mechanisms of action may vary according to the subtype under investigation. In addition, measuring FoxO3a and FoxM1 expression might possibly be a valuable tool to identify sensitive and resistant patients, to stratify groups with different clinical outcomes. We are currently conducting further in vivo experiments in order to confirm the efficacy of LQB-118 in tumor-bearing mice. Taken together, our findings propose the novel pterocarpanquinone LQB-118 compound as a potential future therapeutics for treatment of AML.

\section{Acknowledgements}

We thank Professor Vívian M. Rumjanek for valuable comments on this manuscript. This study was supported by grants from
Instituto Nacional de Ciência e Tecnologia (INCT), Conselho Nacional de Desenvolvimento Científico e Tecnológico (CNPq), Fundação de Amparo à Pesquisa do Rio de Janeiro (FAPERJ), Programa de Oncobiologia and Coordenação de Aperfeiçoamento de Pessoal de Nível Superior (CAPES).

\section{References}

1. Kumar CC: Genetic abnormalities and challenges in the treatment of acute myeloid leukemia. Genes Cancer 2: 95-107, 2011.

2. Bennett JM, Catovsky D, Daniel MT, et al: Proposed revised criteria for the classification of acute myeloid leukemia. A report of the French-American-British Cooperative Group. Ann Intern Med 103: 620-625, 1985.

3. Harris NL, Jaffe ES, Diebold J, et al: World Health Organization classification of neoplastic diseases of the hematopoietic and lymphoid tissues: report of the Clinical Advisory Committee meeting - Airlie House, Virginia, November 1997. J Clin Oncol 17: 3835-3849, 1999.

4. O'Donnell MR, Abboud CN, Altman J, et al: Acute myeloid leukemia. J Natl Compr Cancer Netw 10: 984-1021, 2012.

5. Tallman MS, Nabhan C, Feusner JH, et al: Acute promyelocytic leukemia: evolving therapeutic strategies. Blood 99: 759-767, 2002.

6. Smith ML, Hills RK and Grimwade D: Independent prognostic variables in acute myeloid leukaemia. Blood Rev 25: 39-51, 2011.

7. Wood WA and Lee SJ: Malignant hematologic diseases in adolescents and young adults. Blood 117: 5803-5815, 2011.

8. Shaffer BC, Gillet JP, Patel C, et al: Drug resistance: still a daunting challenge to the successful treatment of AML. Drug Resist Updat 15: 62-69, 2012.

9. Netto CD, Santos ES, Castro CP, et al: (+/-)-3,4-Dihydroxy-8,9methylenedioxypterocarpan and derivatives: cytotoxic effect on human leukemia cell lines. Eur J Med Chem 44: 920-925, 2009.

10. Netto CD, da Silva AJ, Salustiano EJ, et al: New pterocarpanquinones: synthesis, antineoplasic activity on cultured human malignant cell lines and TNF-alpha modulation in human PBMC cells. Bioorg Med Chem 18: 1610-1616, 2010.

11. Maia RC, Vasconcelos FC, de Sá Bacelar T, et al: LQB-118, a pterocarpanquinone structurally related to lapachol [2-hydroxy3-(3-methyl-2-butenyl)-1,4-naphthoquinone]: a novel class of agent with high apoptotic effect in chronic myeloid leukemia cells. Invest New Drugs 29: 1143-1155, 2011.

12. Salustiano EJ, Netto CD, Fernandes RF, et al: Comparison of the cytotoxic effect of lapachol, alpha-lapachone and pentacyclic 1,4-naphthoquinones on human leukemic cells. Invest New Drugs 28: 139-144, 2010.

13. de Sá Bacelar T, da Silva AJ, Costa PR, et al: The pterocarpanquinone LQB 118 induces apoptosis in tumor cells through the intrinsic pathway and the endoplasmic reticulum stress pathway. Anticancer Drugs 24: 73-83, 2013.

14. Adida C, Recher C, Raffoux E, et al: Expression and prognostic significance of survivin in de novo acute myeloid leukaemia. Br J Haematol 111: 196-203, 2000.

15. Tamm I, Kornblau SM, Segall H, et al: Expression and prognostic significance of IAP-family genes in human cancers and myeloid leukemias. Clin Cancer Res 6: 1796-1803, 2000.

16. Wuchter C, Leonid K, Ruppert V, et al: Clinical significance of $\mathrm{P}$-glycoprotein expression and function for response to induction chemotherapy, relapse rate and overall survival in acute leukemia. Leukemia 14: 1018-1024, 2000.

17. Nestal de Moraes G, Silva KL, Vasconcelos FC, et al: Survivin overexpression correlates with an apoptosis-resistant phenotype in chronic myeloid leukemia cells. Oncol Rep 25: 1613-1619, 2011.

18. Nestal de Moraes G, Souza PS, Costas FC, et al: The interface between BCR-ABL-dependent and -independent resistance signaling pathways in chronic myeloid leukemia. Leuk Res Treatment 2012: 671702, 2012.

19. Silva KL, de Souza PS, Nestal de Moraes G, et al: XIAP and P-glycoprotein co-expression is related to imatinib resistance in chronic myeloid leukemia cells. Leuk Res 37: 1350-1358, 2013.

20. Souza PS, Vasconcelos FC, De Souza Reis FR, et al: P-glycoprotein and survivin simultaneously regulate vincristine-induced apoptosis in chronic myeloid leukemia cells. Int J Oncol 39: 925-933, 2011.

21. de Souza Reis FR, de Faria FC, Castro CP, et al: The therapeutical potential of a novel pterocarpanquinone LQB-118 to target inhibitor of apoptosis proteins in acute myeloid leukemia cells. Anticancer Agents Med Chem 13: 341-351, 2013. 
22. Myatt SS and Lam EW: The emerging roles of forkhead box (Fox) proteins in cancer. Nat Rev Cancer 7: 847-859, 2007.

23. Chapuis N, Park S, Leotoing L, et al: IкB kinase overcomes PI3K/Akt and ERK/MAPK to control FoxO3a activity in acute myeloid leukemia. Blood 116: 4240-4250, 2010.

24. Yang JY and Hung MC: A new fork for clinical application: targeting forkhead transcription factors in cancer. Clin Cancer Res 15: 752-757, 2009.

25. Zhang X, Tang N, Hadden TJ, et al: Akt, FoxO and regulation of apoptosis. Biochim Biophys Acta 1813: 1978-1986, 2011.

26. Leung TW, Lin SS, Tsang AC, et al: Over-expression of FoxM1 stimulates cyclin B1 expression. FEBS Lett 507: 59-66, 2001.

27. Wang IC, Chen YJ and Hughes D: Forkhead box M1 regulates the transcriptional network of genes essential for mitotic progression and genes encoding the SCF (Skp2-Cks1) ubiquitin ligase. Mol Cell Biol 25: 10875-10894, 2005.

28. Nakamura S, Hirano I, Okinaka K, et al: The FOXM1 transcriptional factor promotes the proliferation of leukemia cells through modulation of cell cycle progression in acute myeloid leukemia. Carcinogenesis 31: 2012-2021, 2010

29. Wilson MS, Brosens JJ, Schwenen HD, et al: FOXO and FOXM1 in cancer: the FOXO-FOXM1 axis shapes the outcome of cancer chemotherapy. Curr Drug Targets 12: 1256-1266, 2011.

30. Gallagher R, Collins S, Trujillo J, et al: Characterization of the continuous, differentiating myeloid cell line (HL-60) from a patient with acute promyelocytic leukemia. Blood 54: 713-733, 1979.

31. Lee KH, Chang MY, Ahn JI, et al: Differential gene expression in retinoic acid-induced differentiation of acute promyelocytic leukemia cells, NB4 and HL-60 cells. Biochem Biophys Res Commun 296: 1125-1133, 2002.

32. Ralph P, Harris PE, Punjabi CJ, et al: Lymphokine inducing 'terminal differentiation' of the human monoblast leukemia line U937: a role for gamma interferon. Blood 62: 1169-1175, 1983.

33. Cáceres-Cortés JR: Blastic leukaemias (AML): a biologist's view. Cell Biochem Biophys 66: 13-22, 2013.

34. Lorin S, Hamaï A, Mehrpour M, et al: Autophagy regulation and its role in cancer. Semin Cancer Biol 23: 361-379, 2013

35. Wrighton KH: Autophagy: kinase crosstalk through beclin 1. Nat Rev Mol Cell Biol 14: 402-403, 2013.

36. Sunters A, Fernández de Mattos S, Stahl M, et al: FoxO3a transcriptional regulation of Bim controls apoptosis in paclitaxeltreated breast cancer cell lines. J Biol Chem 278: 49795-49805, 2003.

37. Kelly RJ, Lopez-Chavez A, Citrin D, et al: Inhibitors of apoptosis proteins (IAPs) as potential molecular targets for therapy of hematological malignancies. Curr Mol Med 11: 633-649, 2011.
38. Howlader N, Noone AM, Krapcho M, et al: SEER Cancer Statistics Review, 1975-2010, National Cancer Institute. Bethesda, MD, http://seer.cancer.gov/csr/1975 2010/, based on November 2012 SEER data submission, posted to the SEER web site, 2013.

39. da Cunha-Júnior EF, Pacienza-Lima W, Ribeiro GA, et al Effectiveness of the local or oral delivery of the novel naphthopterocarpanquinone LQB-118 against cutaneous leishmaniasis. J Antimicrob Chemother 66: 1555-1559, 2011.

40. Ribeiro GA, Cunha-Júnior EF, Pinheiro RO, et al: LQB-118, an orally active pterocarpanquinone, induces selective oxidative stress and apoptosis in Leishmania amazonensis. J Antimicrob Chemother 68: 789-799, 2013.

41. Van Der Heide LP, Hoekman MF and Smidt MP: The ins and outs of FoxO shuttling: mechanisms of FoxO translocation and transcriptional regulation. Biochem J 380: 297-309, 2004.

42. Santamaría CM, Chillón MC, García-Sanz R, et al: High FoxO3a expression is associated with a poorer prognosis in AML with normal cytogenetics. Leuk Res 33: 1706-1709, 2009.

43. Sykes SM, Lane SW, Bullinger L, et al: AKT/FOXO signaling enforces reversible differentiation blockade in myeloid leukemia. Cell 146: 697-708, 2011.

44. Kornblau SM, Singh N, Qiu Y, et al: Highly phosphorylated FOXO3A is an adverse prognostic factor in acute myeloid leukemia. Clin Cancer Res 16: 1865-1874, 2010.

45. Sakoe Y, Sakoe K, Kirito K, et al: FOXO3A as a key molecule for all-trans retinoic acid-induced granulocytic differentiation and apoptosis in acutepromyelocytic leukemia. Blood 115: 3787-3795, 2010

46. Thépot S, Lainey E, Cluzeau T, et al: Hypomethylating agents reactivate FOXO3A in acute myeloid leukemia. Cell Cycle 10 2323-2330, 2011.

47. Kelly KR, Nawrocki ST, Espitia CM, et al: Targeting Aurora A kinase activity with the investigational agent alisertib increases the efficacy of cytarabine through a FOXO-dependent mechanism. Int J Cancer 131: 2693-2703, 2012

48. Scheijen B, Ngo HT, Kang H, et al: FLT3 receptors with internal tandem duplications promote cell viability and proliferation by signaling through Foxo proteins. Oncogene 23: 3338-3349, 2004.

49. Zhao F and Lam EW: Role of the forkhead transcription factor FOXO-FOXM1 axis in cancer and drug resistance. Front Med 6: 376-380, 2012

50. Koo CY, Muir KW and Lam EW: FOXM1: from cancer initiation to progression and treatment. Biochim Biophys Acta 1819: 28-37, 2012.

51. Zhang X, Zeng J, Zhou M, et al: The tumor suppressive role of miRNA-370 by targeting FoxM1 in acute myeloid leukemia. Mol Cancer 11: 56, 2012. 